\title{
Sheep sclera soft tissue subjected to mechanical equi-biaxial testing
}

\author{
Zwelihle Ndlovu', ${ }^{1,2}$, Dawood Desia ${ }^{2}$, Harry Ngwangwa ${ }^{1}$, Fulufhelo Nemavhola ${ }^{1 *}$
}

1 Unisa Biomechanics Lab, Department of Mechanical and Industrial Engineering, School of Engineering, College of Science Engineering and Technology, University of South Africa, Unisa Science Campus, Johannesburg, South Africa; masitfj@unisa.ac.za

2 Department of Mechanical and Mechatronics Engineering, Faculty of Engineering and Built Environment, Tshwane University of Technology; DesaiDA@tut.ac.za

* Correspondence: masitfj@unisa.ac.za ; Tel.: (+27 (011) 471 2354)

\begin{abstract}
A better understanding of diseases progress in tissues vest on the accurate understanding of tissues under mechanical loading. Also, development of therapies for injuries may depend on the available mechanical data for soft tissues. In this study, the raw data of biaxial tensile testing of sclera soft tissue is presented in this paper. Biaxial mechanical testing of soft tissues presents details understanding of how soft tissues behave when compared to uniaxial testing. Biomechanical properties of soft tissues are vital in the development of accurate computational models. Reliable computational models of studying mechanisms of diseases depends mainly on the accurate and more details mechanical behavior of soft tissues. These accurate and detailed computational models may be utilized to further develop the understanding and therapies of various diseases. The mechanical tensile testing was conducted on the passive sheep sclera. Engineering stress vs strain of several samples of the sheep sclera are further presented determined from force and displacement experimental data. The goal of this paper is to make available biaxial data of sheep sclera soft tissue that can be further utilized.
\end{abstract}

Dataset: https://doi.org/10.17632/3xb9hkny9f.2

Dataset License: license under which the dataset is made available (CC-BY.)

Keywords: Sclera tissue; biaxial testing; soft tissue mechanics; mechanical properties

\section{Summary}

Biomechanical properties of soft tissue have been utilized to study the mechanisms of various diseases. The mechanics of sclera is vital in studying the mechanisms of eye diseases such as glaucoma. The dataset presented here is useful in biomechanics of sclera soft tissue of a sheep animal. Understanding modalities of eye diseases is critical in the development of medical therapies. The mechanical data may be utilised as an animal model to study various mechanisms of eye diseases. Also, the dataset is useful to researcher communities who has interest in study various mechanisms of the eye. In addition, the data may be use/re-used by developing computational models to study various diseases of the eye by computing the mechanical constants of the sclera tissue subjected to biaxial tensile testing. Biaxial mechanical properties dataset may provide more details when compared to uniaxial testing. Unlike what has been commonly presented in various studies, here, we present the biaxial mechanical properties of sclera soft tissue of the sheep. 


\section{Data Description}

The shared data shows the mechanical properties of various sheep sclera tissue. The biaxial stress-strain tensile data (in both longitudinal and circumferential directions) of sclera tissue are shown in Figure 1 (a)-(k). Figure 4 shows the maximum tensile stress at maximum strain of sheep sclera subjected to equi-biaxial force before failure.
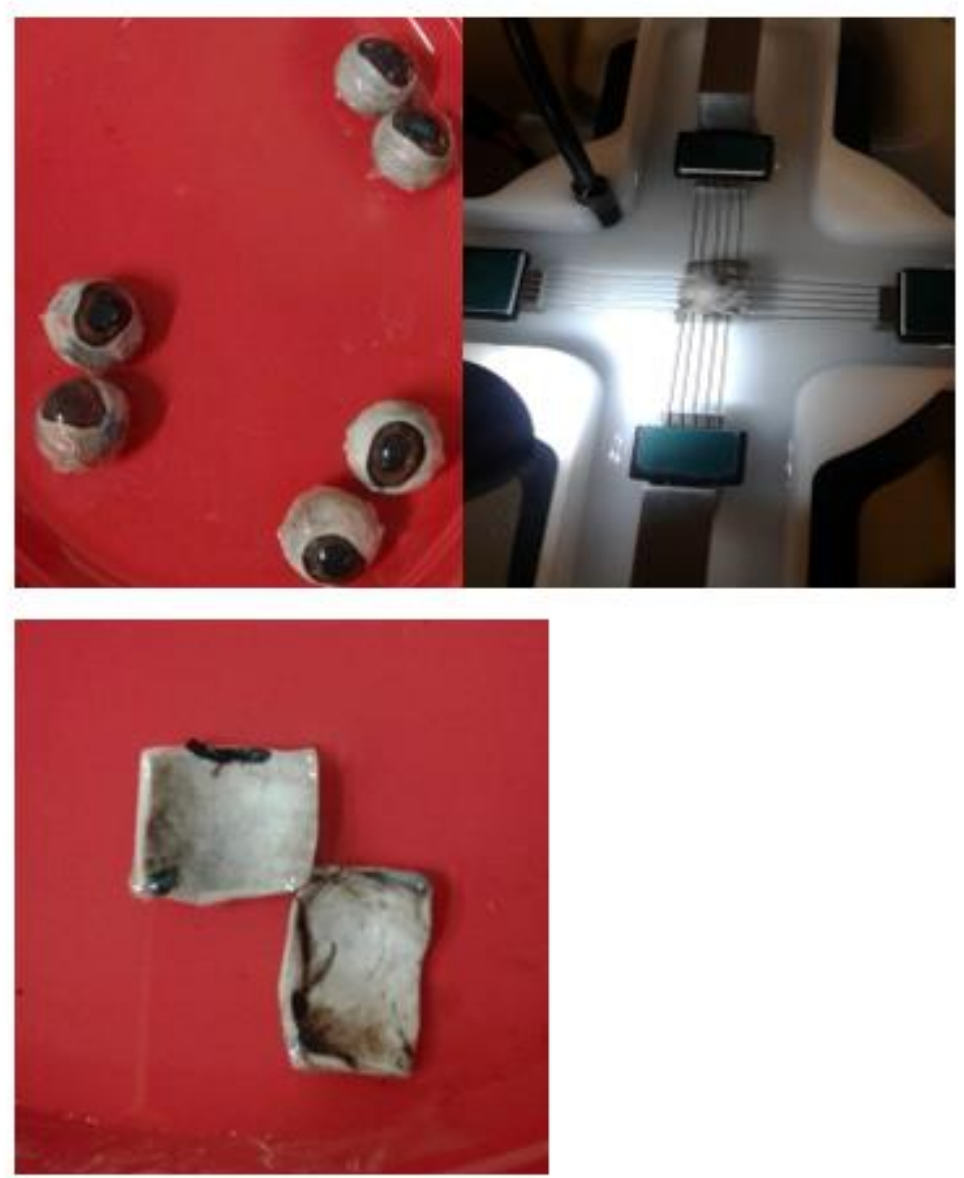

Figure 1. Experimental set-up of biaxial testing of sheep sclera soft tissue.

\section{Experimental Design, Materials and Methods \\ 3.1 Specimen (Sclera soft tissue) preparation}

Eleven $(\mathrm{N}=11)$ fresh eyes of unknown age with no known diseases related to eyes were collected from the local abattoir. Immediately after 2 hours after slaughter, the eyes were placed in ice box for delivery to the Unisa biomechanics Lab for testing. The mechanical testing took place between 3 and 6 hours after harvest. To reduce the thickness variation of the sclera tissue, $12 \times 12 \mathrm{~mm}$ squares specimen was cut from each eye. The cut was done approximately $6 \mathrm{~mm}$ from the optic nerve head and corneal limbus. To measure the thickness of the $12 \times 12 \mathrm{~mm}$, the Vernier caliper was utilized to measure thickness at four places (Figure 1). The four measurements that are taken, are the averaged for further processing in determining the cross-sectional Area $\left(\mathrm{A}_{\mathrm{i}}\right)$. The specimens were then subjected to equibiaxial testing. Longitudinal $(\mathrm{Y})$ direction points towards the lens and regard as $0 \mathrm{o}$ and the circumferential was measure 90o from the longitudinal direction $(\mathrm{Y})$. To maintain and refresh (muscle relaxation) the tissue, the specimens were dipped and kept in isotonic solution $9.0 \mathrm{~g} / \mathrm{l}$ at $\mathrm{pH}$ of 5.5 for about 15 to 20 minutes before testing. Specimen were tested at $\left(37^{\circ} \mathrm{C}\right)$ to mimic body temperature. 


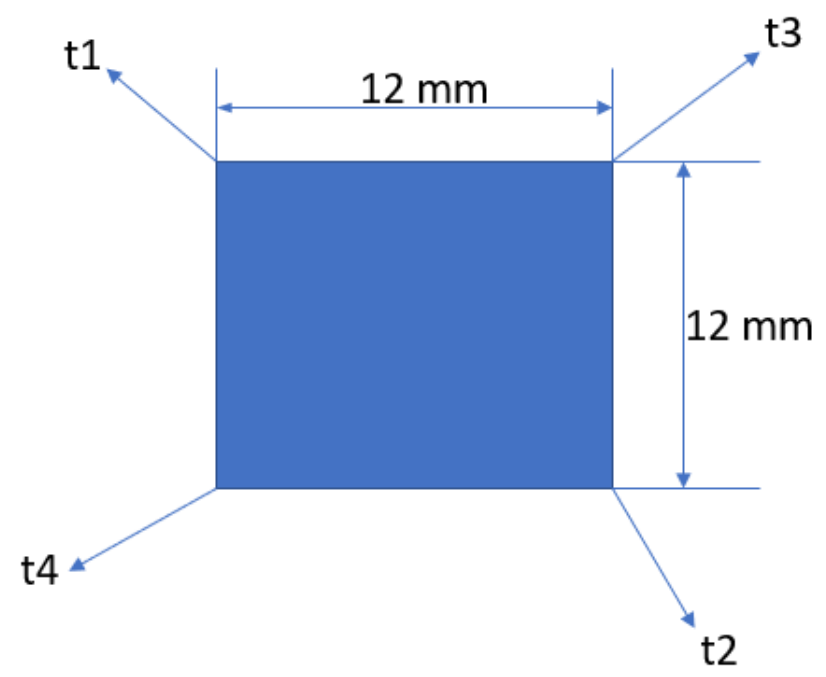

Figure 1: Measurement of thickness in the harvested specimen of $12 \mathrm{~mm}$. The four measurements that are taken, are the averaged for further processing in determining the cross-sectional Area $\left(A_{i}\right)$

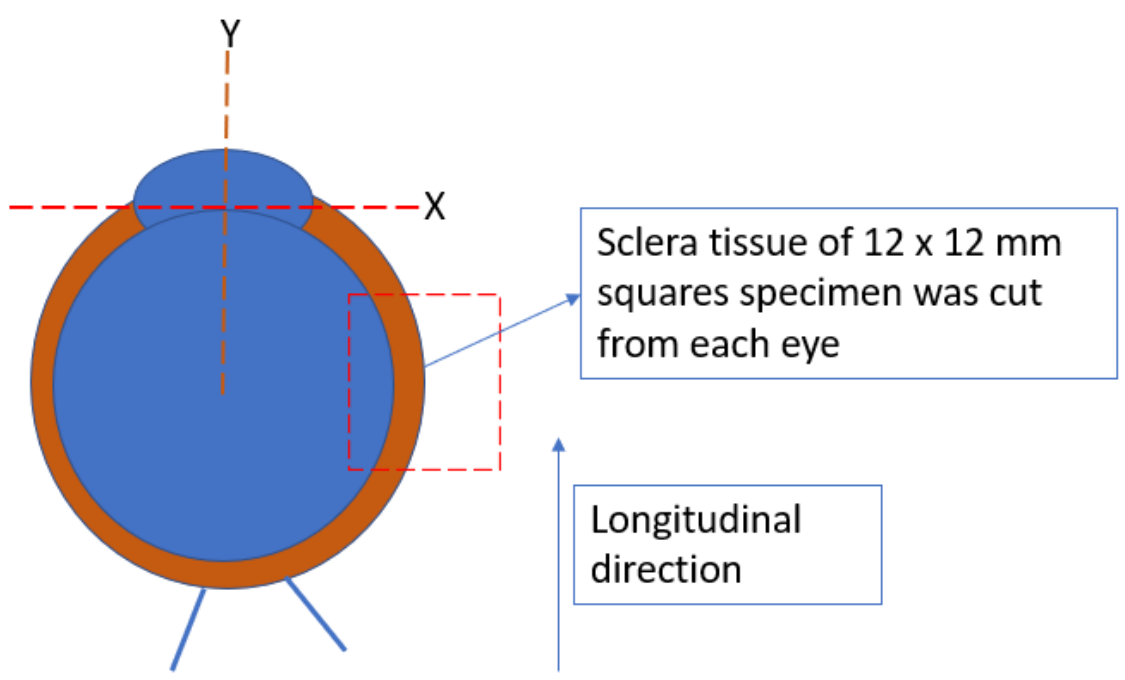

Figure 2: Cutting of $12 \times 12 \mathrm{~mm}$ sclera tissue from the eye collected from the abattoir. Longitudinal (Y) direction points towards the lens and regard as $0^{\circ}$ and the circumferential was measure $90^{\circ}$ from the longitudinal direction $(\mathrm{Y})$.

\subsection{Mechanical testing}

Biomechanical properties of the sclera tissue of the sheep were captured by subjecting the $12 \times 12 \mathrm{~mm}$ square sample to equi-biaxial forces. This was achieved by utilising the BioTester 5000 from Cellscale (Waterloo, Canada). The BioTester 5000 has a load capacity of $23 \mathrm{~N}$ and accuracy of (+/-) $5 \mathrm{mN}$. Strain ranges of 0.0133 and $0.89 \mathrm{~mm} / \mathrm{s}$ were used, and the force and displacement data were collected at $30 \mathrm{~Hz}$. Pretension of $50 \mathrm{mN}$ was applied during testing and the preconditioning was achieved by applying 10 cycles of equi-biaxial stretch at a speed of $0.5 \mathrm{~mm} / \mathrm{s}$. Engineering stress $(\sigma=F / A)$ and Engineering strain $\left(\varepsilon=\Delta l / l_{o}\right)$ were calculated from the force and displacement data of the equi-biaxial test. Where Area is calculated from $A=t_{i} x b$ b is the breath of the specimen and $\mathrm{t}$ is the average thickness measured by the Vernier Calliper ( $t_{i}=$ $\left(t_{1}+t_{2}+t_{3}+t_{4} / 4\right)$. 
Table 1: Measurement of thickness of the sclera soft tissue measured as shown in Figure 1.

\begin{tabular}{|l|r|r|r|r|r|}
\hline & Thickness $\left(\mathrm{t}_{1}\right)$ & \multicolumn{1}{|c|}{ Thickness $\left(\mathrm{t}_{2}\right)$} & \multicolumn{1}{|c|}{ Thickness $\left(\mathrm{t}_{3}\right)$} & Thickness $\left(\mathrm{t}_{4}\right)$ & Average \\
\hline Specimen 1 & 1.12 & 1.13 & 0.96 & 0.87 & 1.02 \\
\hline Specimen 2 & 1.02 & 0.96 & 0.88 & 1.20 & 1.02 \\
\hline Specimen 3 & 0.96 & 0.88 & 0.94 & 1.13 & 0.98 \\
\hline Specimen 4 & 1.01 & 0.76 & 1.10 & 1.04 & 0.98 \\
\hline Specimen 5 & 1.20 & 0.96 & 0.87 & 0.96 & 1.00 \\
\hline Specimen 6 & 0.85 & 1.10 & 1.16 & 0.83 & 0.99 \\
\hline Specimen 7 & 0.95 & 1.02 & 1.12 & 1.11 & 1.05 \\
\hline Specimen 8 & 0.89 & 1.09 & 1.42 & 1.12 & 1.13 \\
\hline Specimen 9 & 0.97 & 1.14 & 0.96 & 1.05 & 1.03 \\
\hline Specimen 10 & 0.94 & 1.16 & 0.98 & 1.08 & 1.04 \\
\hline Specimen 11 & 1.24 & 1.12 & 0.85 & 1.14 & 1.09 \\
\hline Average & $\mathbf{1 . 0 1}$ & $\mathbf{1 . 0 3}$ & $\mathbf{1 . 0 2}$ & $\mathbf{1 . 0 5}$ & $\mathbf{1 . 0 3}$ \\
\hline
\end{tabular}

\section{Results}

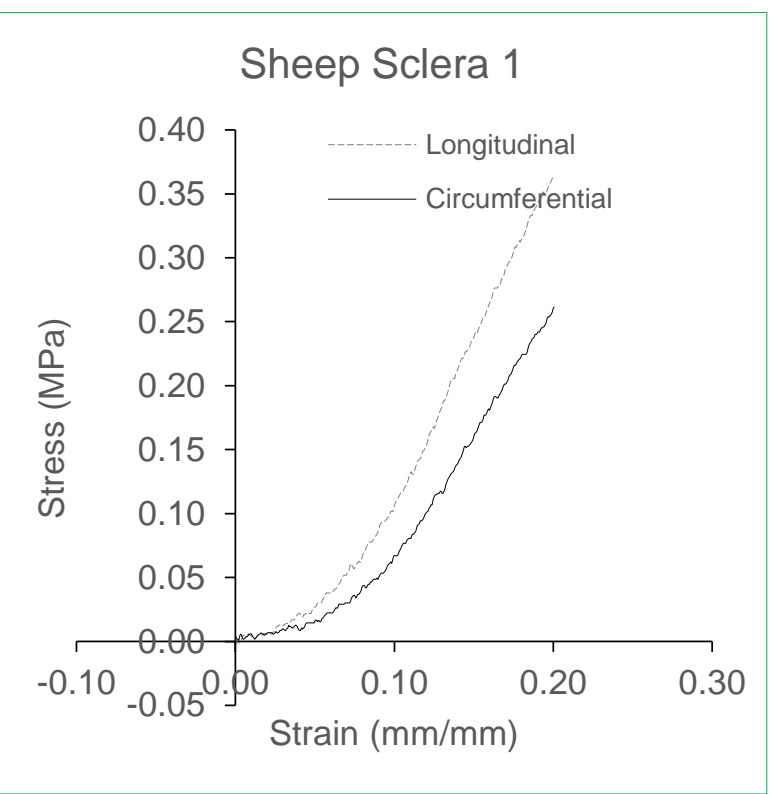

(a)

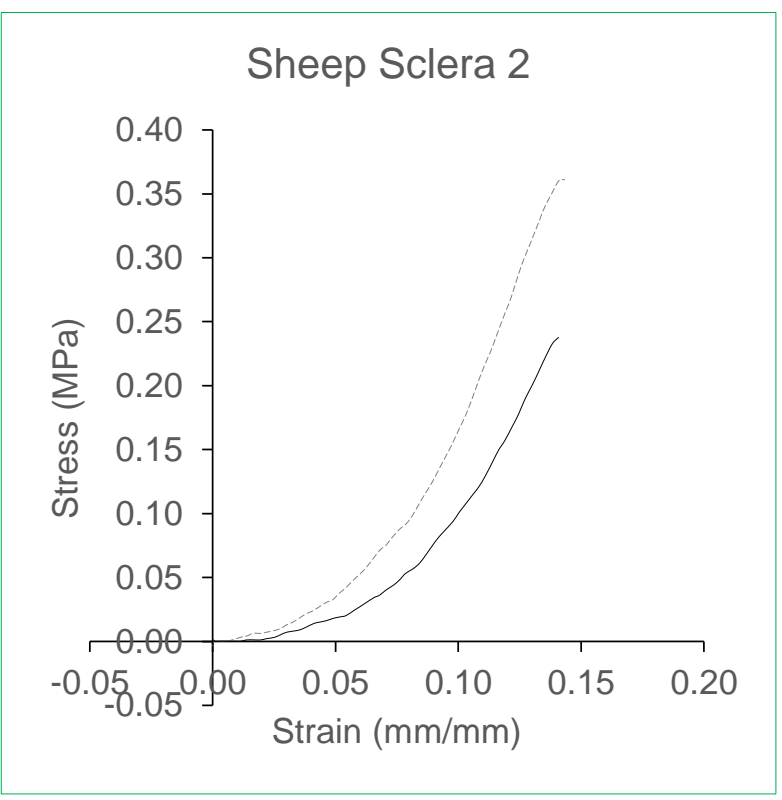

(b) 


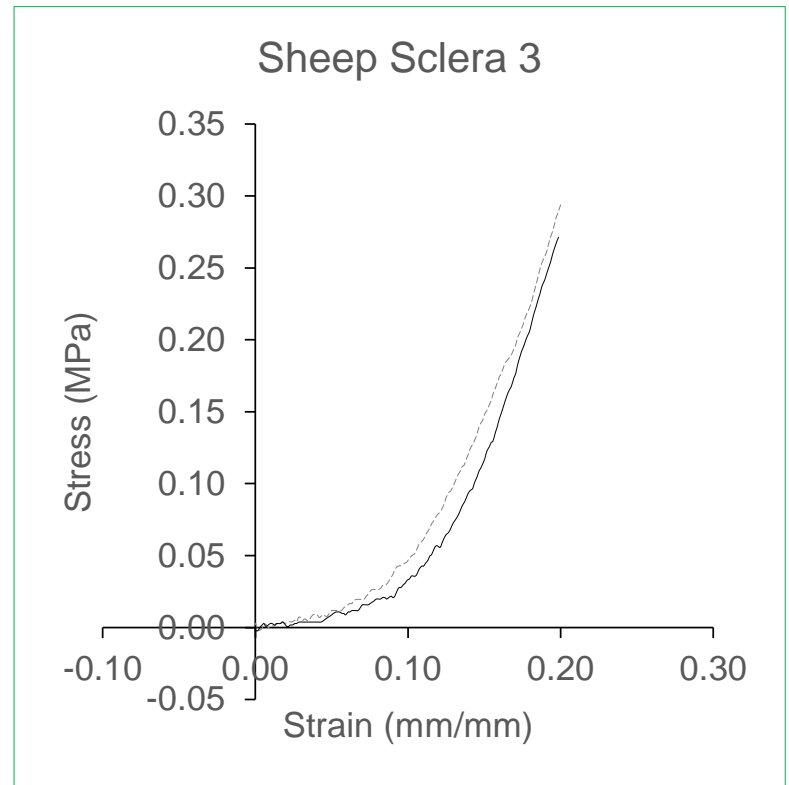

(c)

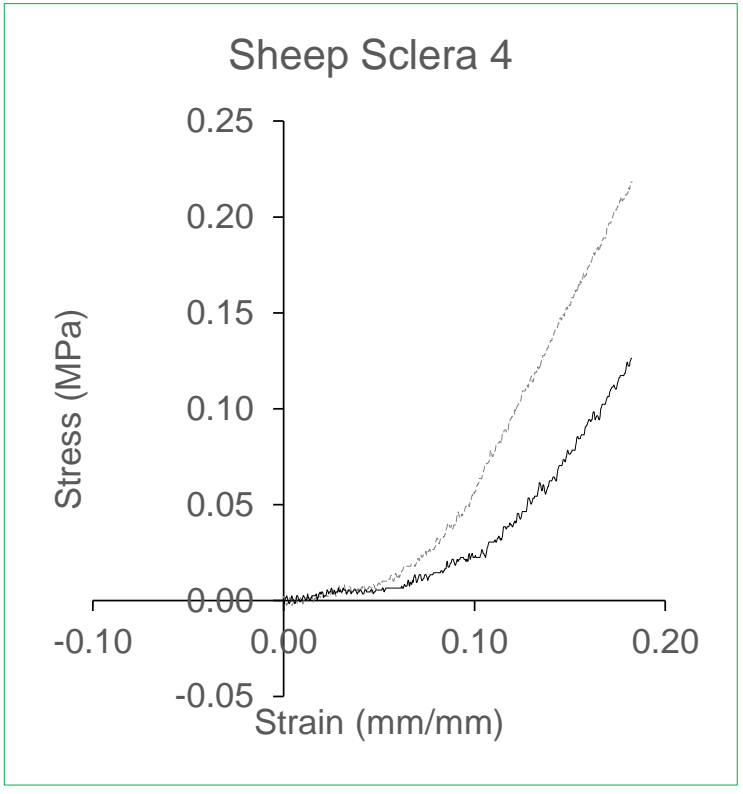

(d)

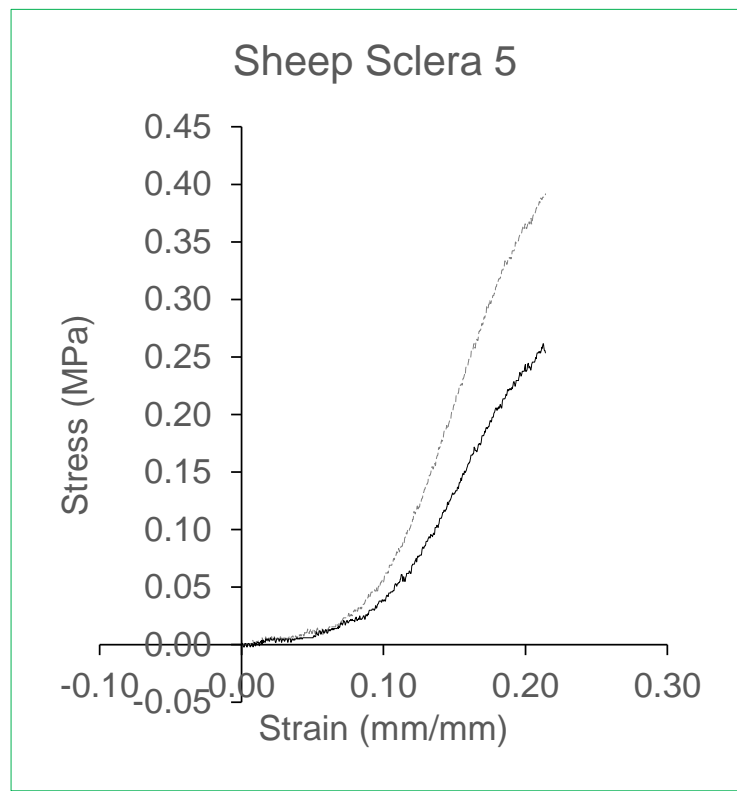

(e)

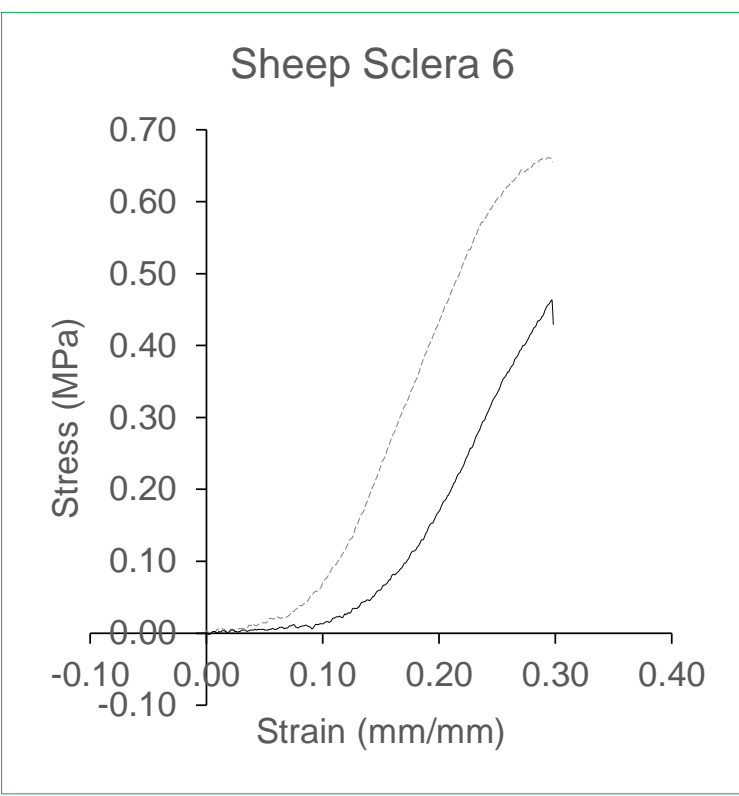

(f) 


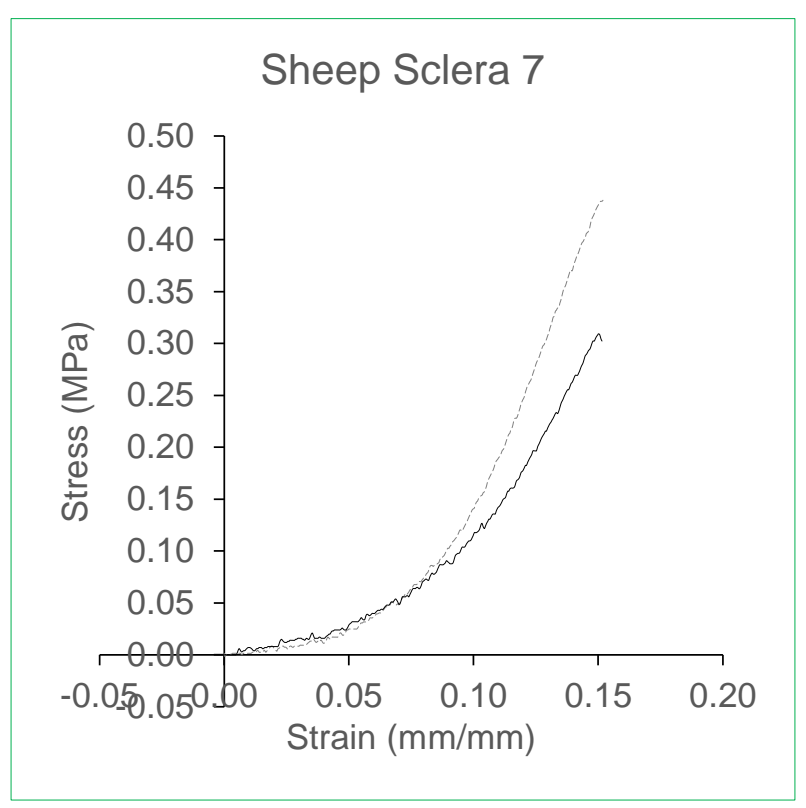

(g)

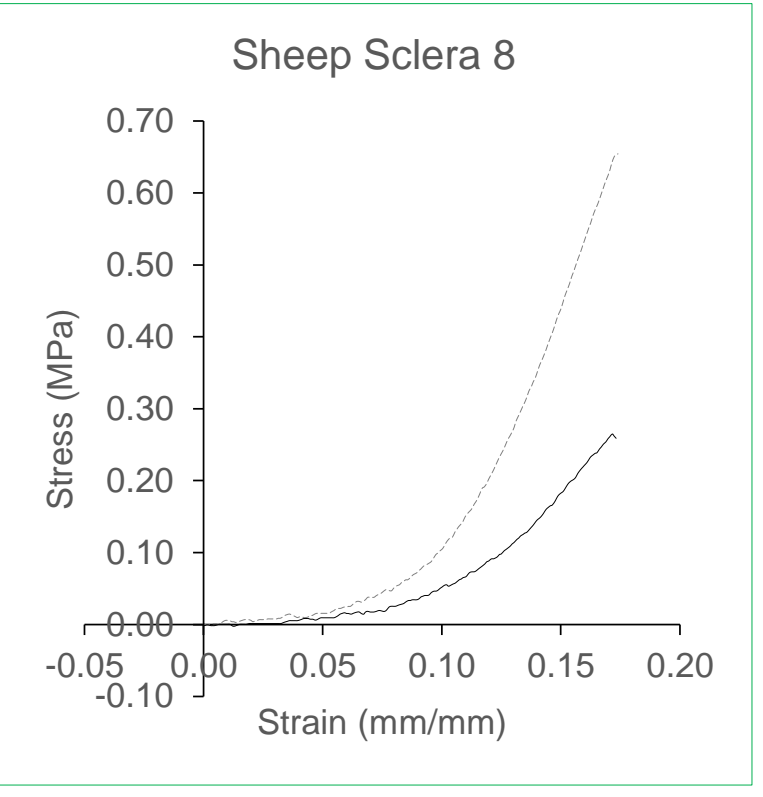

(i)

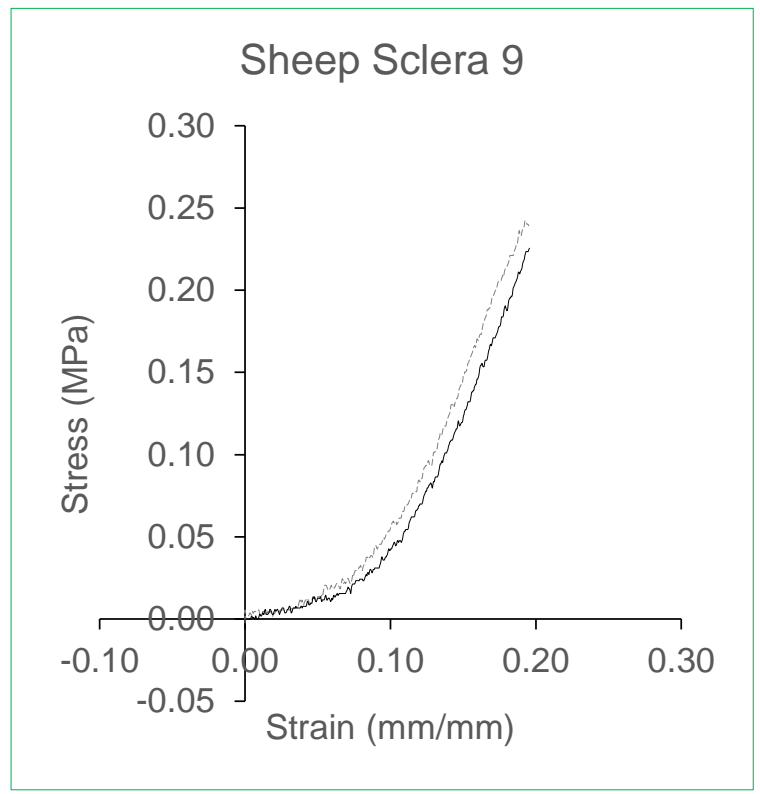

(i)

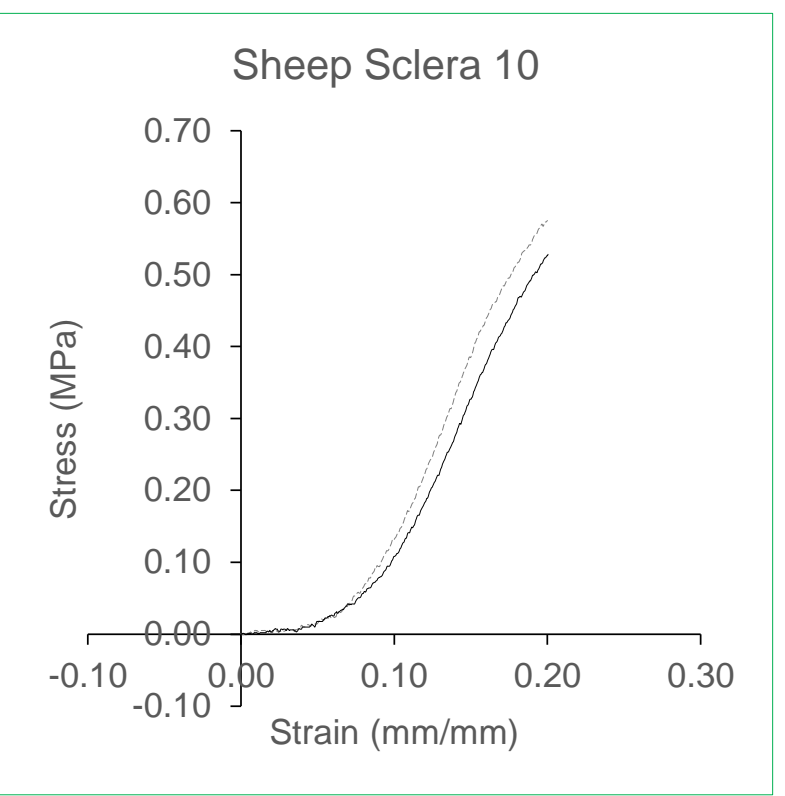

(j) 


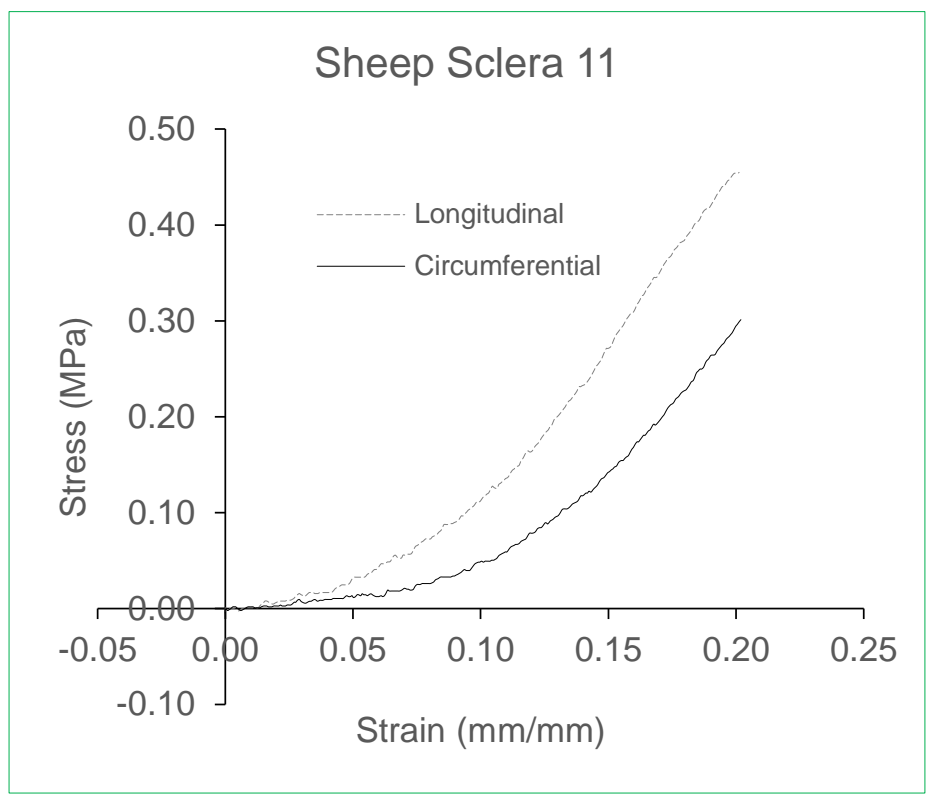

(k)

Figure 3: Stress-strain of sheep sclera soft tissue under equi-biaxial tensile testing. Stress and strain in the longitudinal and circumferential directions were calculated from the force and displacement data.

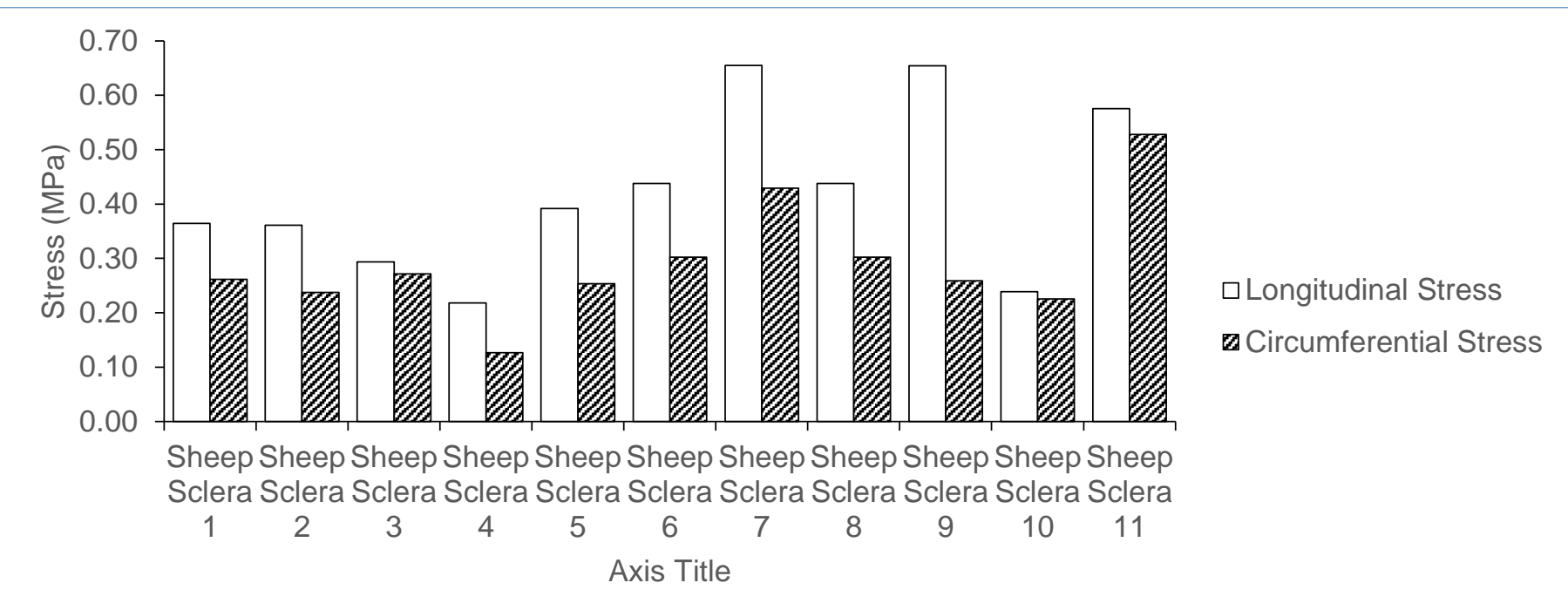

Figure 4: Stress at maximum strain in both longitudinal and circumferential direction during biaxial tensile testing of the sheep sclera 


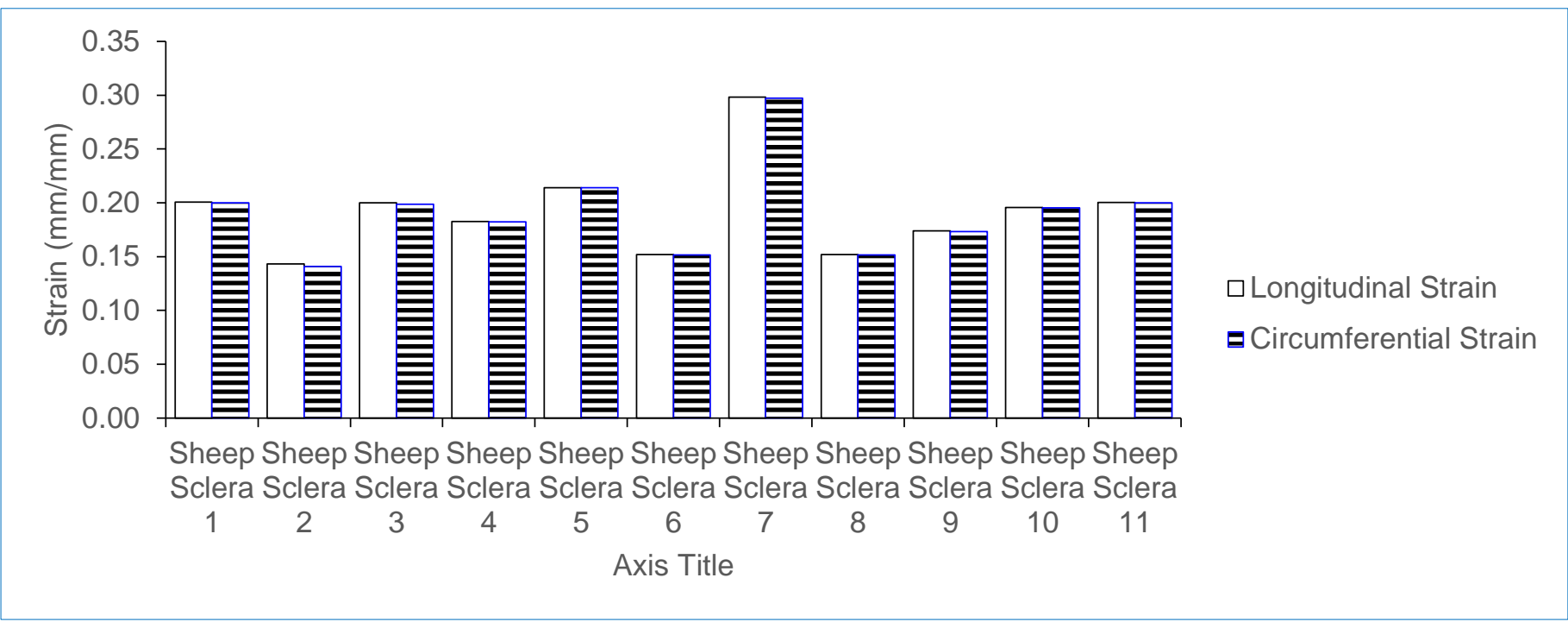

Figure 5: Maximum strain in both longitudinal and circumferential direction during biaxial tensile testing of the sheep sclera

\section{Discussion}

Accurate biomechanical properties of soft tissues plays an important role in understanding diseases [1-4]. This understanding maybe achieved by further developing the constitutive models of the soft tissue. The constitutive models require accurate material parameters for the development of computational models. Computational models have been utilized to understand various mechanisms of diseases[5-10]. In this study, the equi-biaxial mechanical properties of the sheep sclera are presented. This data was used to further determine the material parameters of various constitutive models [11].

Supplementary Materials: No supplementary data is available except data that is available here https://doi.org/10.17632/3xb9hkny9f.2

Author Contributions: Conceptualization, F.N.; methodology, F.N.; formal analysis, Z.N.,F.N. and D.D.; investigation, F.N., H.M, D.D. and Z.N.; resources, F.N. and H.N.; data curation, Z.N and F.N.; writing - original draft preparation, F.N.; writing - review and editing, H.N. D.D and Z.N.; visualization, F.N.; supervision, F.N, D.D and H.N.; project administration, H.M. and F.N.; funding acquisition, F.N. and H.N., All authors have read and agreed to the published version of the manuscript.

Funding: Support from the National Research Foundation (NRF) Grant number (129380)

Data Availability Statement: The dataset presented in this paper can be found publicly deposited here https://doi.org/10.17632/3xb9hkny9f.2

Acknowledgments: The authors would like to acknowledge the National Research Foundation (NRF) Gran number (129380) is gratefully acknowledged. Unisa CAPEX Programme supported the acquisition of biaxial testing machine in the Department of Mechanical Engineering, School of Engineering, College of Science Engineering and Technology. We also acknowledge graduate researchers Mr Lebogang Lebea and Ms Lebogang Mathebela for their assistance with the biaxial mechanical testing.

Conflicts of Interest: The authors declare no conflict of interest. The funders had no role in the design of the study; in the collection, analyses, or interpretation of data; in the writing of the manuscript, or in the decision to publish the results.

\section{References}

1. Ngwangwa, H.M. and F. Nemavhola, Evaluating computational performances of hyperelastic models on supraspinatus tendon uniaxial tensile test data. Journal of Computational Applied Mechanics, 2021. 52(1): p. 27-43.

2. Nemavhola, F., Biaxial quantification of passive porcine myocardium elastic properties by region. Engineering Solid Mechanics, 2017. 5(3): p. 155-166. 
3. Nemavhola, F., Study of biaxial mechanical properties of the passive pig heart: material characterisation and categorisation of regional differences. International Journal of Mechanical and Materials Engineering, 2021. 16(1): p. 1-14.

4. Nemavhola, F., et al., Passive Biaxial Tensile Dataset of Three Main Rat Heart Myocardia: Left Ventricle, Mid-Wall and Right Ventricle. Preprints, 2021. 2021080153(Version 1).

5. Nemavhola, F., Detailed structural assessment of healthy interventricular septum in the presence of remodeling infarct in the free wall-A finite element model. Heliyon, 2019. 5(6): p. e01841.

6. Nemavhola, F., Mechanics of the septal wall may be affected by the presence of fibrotic infarct in the free wall at end-systole. International Journal of Medical Engineering and Informatics, 2019. 11(3): p. 205-225.

7. $\quad$ Masithulela, F.J., Computational biomechanics in the remodelling rat heart post myocardial infarction. 2016.

8. Masithulela, F., Bi-ventricular finite element model of right ventricle overload in the healthy rat heart. Bio-medical materials and engineering, 2016. 27(5): p. 507-525.

9. Masithulela, F. The effect of over-loaded right ventricle during passive filling in rat heart: A biventricular finite element model. in ASME International Mechanical Engineering Congress and Exposition. 2015. American Society of Mechanical Engineers.

10. Masithulela, F. Analysis of passive filling with fibrotic myocardial infarction. in ASME international mechanical engineering congress and exposition. 2015. American Society of Mechanical Engineers.

11. Ndlovu, Z., F. Nemavhola, and D. Desai, BIAXIAL MECHANICAL CHARACTERIZATION AND CONSTITUTIVE MODELLING OF SHEEP SCLERA SOFT TISSUE. Russian Journal of Biomechanics/Rossijski Zurnal Biomehaniki, 2020. 24(1). 